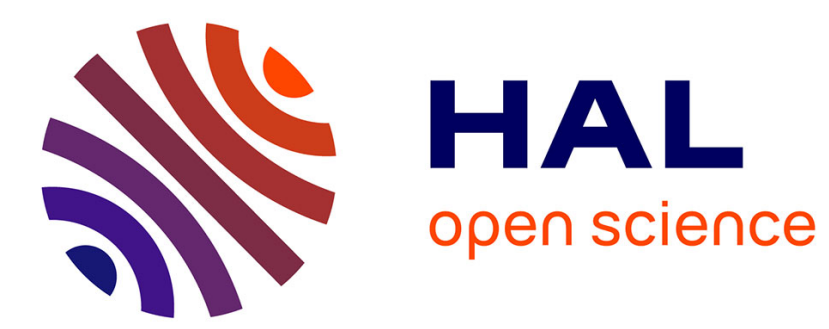

\title{
Fault Diagnosis with Bayesian Networks: Application to the Tennessee Eastman Process
}

Sylvain Verron, Teodor Tiplica, Abdessamad Kobi

\section{To cite this version:}

Sylvain Verron, Teodor Tiplica, Abdessamad Kobi. Fault Diagnosis with Bayesian Networks: Application to the Tennessee Eastman Process. IEEE International Conference on Industrial Technology, 2006, Mumbai, India. inria-00517005

\section{HAL Id: inria-00517005 https://hal.inria.fr/inria-00517005}

Submitted on 13 Sep 2010

HAL is a multi-disciplinary open access archive for the deposit and dissemination of scientific research documents, whether they are published or not. The documents may come from teaching and research institutions in France or abroad, or from public or private research centers.
L'archive ouverte pluridisciplinaire HAL, est destinée au dépôt et à la diffusion de documents scientifiques de niveau recherche, publiés ou non, émanant des établissements d'enseignement et de recherche français ou étrangers, des laboratoires publics ou privés. 


\title{
Fault Diagnosis with Bayesian Networks: Application to the Tennessee Eastman Process
}

\author{
Sylvain VERRON, Teodor TIPLICA, Abdessamad KOBI \\ Laboratoire en Sûreté de Fonctionnement, Qualité et Organisation (LASQUO) \\ ISTIA \\ 62, Avenue Notre Dame du Lac, Angers, 49000 ,France \\ sylvain.verron@istia.univ-angers.fr \\ http: //www . univ-angers. fr/laboratoire. asp? ID=34\&langue=1
}

\begin{abstract}
The purpose of this article is to present and evaluate the performance of a new procedure for industrial process diagnosis. This method is based on the use of a bayesian network as a classifier. But, as the classification performances are not very efficient in the space described by all variables of the process, an identification of important variables is made. This feature selection is made by computing the mutual information between each process variable and the class variable. The performances of this method are evaluated on the data of a benchmark problem: the Tennessee Eastman Process. Three kinds of faults are taken into account on this complex process. The objective is to obtain the minimal recognition error rate for these 3 faults. Results are given and compared with results of other authors on the same data.
\end{abstract}

\section{INTRODUCTION}

Nowadays, industrial processes are more and more complex and are very difficult to control. As a consequence, many sensors are integrated in industrial processes to monitor them. So, we can obtain a large amount of data from the process. In this context, the process control is an essential task. The final goal of the process control is to reduce variability, and so, to improve the quality of the product [1]. The process control comprises four procedures: fault detection (decide if the process is under normal condition or out-of-control); fault identification (identify the variables implicated in an observed out-of-control status); fault diagnosis (find the root cause of the disturbance); process recovery (return the process to a normal status).

In literature, we can find a lot of different data-driven techniques for process control. For the fault detection of industrial processes many methods have been submitted: univariate statistical process control (Shewhart charts) [2], [3], multivariate statistical process control $\left(T^{2}\right.$ and $\mathrm{Q}$ charts) [4], [5], and some PCA (Principal Component Analysis) based techniques [6] like Multiway PCA or Moving PCA [7]. An interesting article is [8] in which authors make comparisons between these different techniques. For the fault identification procedure, one of the better statistical techniques is the MYT decomposition of the $T^{2}$ statistic [9], [10]. Finally, for the fault diagnosis techniques we can cite the book of Chiang, Russell and Braatz [11] which presents a lot of them (PCA based techniques, Fisher Discriminant Analysis, PLS based techniques, etc).

In this article, we present a new procedure for the di- agnosis of the faults of an industrial system based on the utilization of mutual information and bayesian network. The mutual information is used for the selection of the key variables of the system, and bayesian networks are used for classification in the space of the key variables. The article is structured in the following manner. In the second section, we introduce bayesian network $(\mathrm{BN})$ classifiers and some notions concerning the mutual information. In the third section, we present the new diagnosis procedure that we are proposing, using bayesian network and mutual information, to diagnosis the fault of an industrial system. The fourth section presents an application of this procedure for the diagnosis of three types of fault on the benchmark problem Tennessee Eastman Process. Finally, in the last section, we conclude on the advantages and drawbacks of this procedure, and we present outlooks of fault diagnosis with bayesian network classifiers.

\section{BAYESIAN CLASSIFIERS AND MUTUAL INFORMATION}

\section{A. Classification task}

Once a problem (fault) has been detected in the evolution of the process by the mean of a detection method, we need to identify (diagnosis) the belonging class of this fault. Thereby, the diagnosis problem can be viewed as the task to correctly classify this fault in one of the predefined fault classes. The classification task needs the construction of a classifier (a function allocating a class to some observations described by some variables of the system). Two types of classification exist: unsupervised classification which objective is to identify the number and the composition of each class present in the data structure; supervised classification where the number of classes and the belonging class of each observation are known in a learning sample and whose objective is to class new observations to one of the existing classes.

In the case of industrial system diagnosis, we can frequently have a database containing recorded observations of previous faults of the system. So, we can easily use supervised classification to classify a new faulty observation. In this article, we decided to use one of these supervised classification tools, precisely the bayesian network. 


\section{B. Bayesian network classifiers}

A bayesian network (BN) [12], [13], [14] is a graph. In this graph, each variable is a node that can be continuous or discrete. Edges of the graph represent dependence between linked nodes.

A bayesian network is a triplet $\{\mathbf{G}, \mathbf{E}, \mathbf{D}\}$ where: $\{\boldsymbol{G}\}$ is a directed acyclic graph, $\mathbf{G}=(V, A)$, where $V$ is the ensemble of nodes of $\mathbf{G}$, and $A$ is the ensemble of edges of $\mathbf{G}$,

$\{\boldsymbol{E}\}$ is a finite probabilistic space $(\Omega, Z, p)$, where $\Omega$ is a non-empty space, $Z$ is a collection of subspace of $\Omega$, and $p$ is a probability measure on $Z$ with $p(\Omega)=1$,

$\{\boldsymbol{D}\}$ is an ensemble of random variables associated to the nodes of $\mathbf{G}$ and defined on $\mathbf{E}$ such as:

$$
p\left(V_{1}, V_{2}, \ldots, V_{n}\right)=\prod_{i=1}^{n} p\left(V_{i} \mid C\left(V_{i}\right)\right)
$$

where $C\left(V_{i}\right)$ is the ensemble of causes (parents) of $V_{i}$ in the graph $\mathbf{G}$.

Bayesian network classifiers are particular bayesian networks [15], [16]. They always have a discrete node $C$ which codes the $r$ different classes. The other nodes $\left(X_{1}, \ldots, X_{p}\right\}$ represent the $p$ descriptors (variables) of the classification task. These descriptors can be either discrete or continuous variables.

A particular type of BN classifier, used in the context of industrial systems, is the Naïve Bayesian Network (NBN) [17], [16], also known as the Bayes classifier. A NBN is composed of a "class node" representing the different classes of our system. This nodes is linked with all other variables of the system (descriptors) as indicated on the figure 1 .

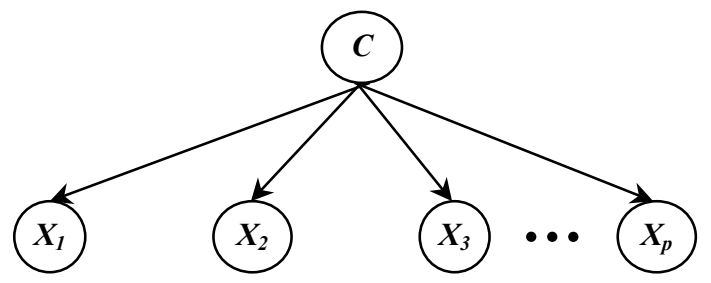

Fig. 1. Naïve bayesian network

Inza [18] made a comparative study of classification algorithms used in artificial intelligence. The performance of NBN were compared with other methods like hierarchic classification, $\mathrm{k}$ nearest neighborhood, C4.5, decision tree and so on [15], [19]. Yang [20] demonstrated that the NBN (in the case that the independence assumption of the descriptors is verified and that probabilities are estimated with enough precision) is an optimal classifier in term of misclassification rate. This optimality is obtained when continuous variables are discretized in such a way that every distribution can be sufficiently well approximated by discretization. Of course, the discretization of variables is a loss of information because it is a reduction of the variables space. But, in a bayesian network, we can have non-discretized continuous variables under the assumption that these variables have a normal distribution. This is the case in many industrial systems. Parameters of these continuous variables must be estimated from data. Two different techniques for parameters estimation are available, the Maximum Likelihood Estimation (MLE) and the bayesian approach. In this paper, the parameters of the network will be estimated by the MLE method. In the case that all descriptors are continuous variables (with normal distribution assumption) and that parameters are estimated with MLE, we obtain the known classifier: diagonal quadratic discriminant function.

A NBN seems to be a good classifier for fault diagnosis in industrial systems [18]. However, a NBN does not take into account the correlations between descriptors which are frequent in industrial systems. As a consequence the classification efficiency is poor in this case. To solve this major problem generated by the correlation between the variables some extensions to the NBN have been developed [16].

A first interesting extension is the TAN (TreeAugmented bayesian Network) [16]. In a TAN, a maximum weighted spanning tree is constructed with the $p$ descriptors following the algorithm of Chow et Liu [21]. So, each descriptor will have at most one other descriptor as parent. After that, edges from the class node to each descriptor are added (like a NBN). An example of a TAN is given on the figure 2 .

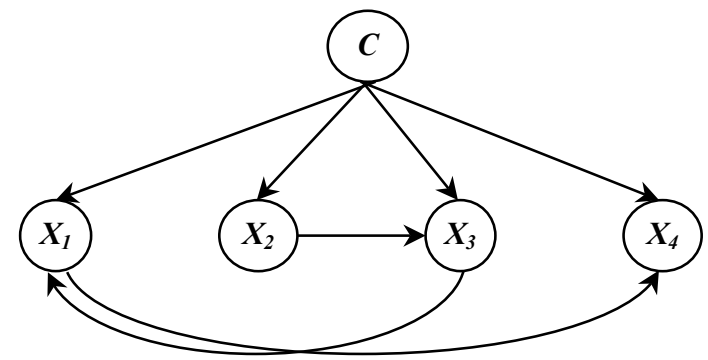

Fig. 2. Tree-Augmented bayesian Network

Another extension is the $\mathrm{k}$-dependence bayesian classifier structure (kDB structure) [22] that extends the TAN structure allowing a maximum of $\mathrm{k}$ predictor parents plus the class for each predictor variable (TAN structures are equivalent to $\mathrm{kDB}$ structures with $\mathrm{k}=1$ ). Finally, the semi naive Bayes structure (Semi structure) introduces joint nodes [23], which are the cartesian product of a subset of original variables. Therefore, every component variable of the joint nodes are mutually statistically dependent.

Of course the TAN classifier, and other extensions, are better than the NBN classifier because they take into account some of the existing correlations between the process variables, but there still remains an important problem to solve. The performances (in term of correct classification rate) of such a classifiers are not so good if non-informative descriptors are present. Actually, if we want to diagnosis 
a system with many variables, only few of them are really important for classification, others (less importants) must not be taken into account. In other words, we have to do a selection of the important variables for the classification task.

\section{Mutual Information for variable selection}

In the information theory, the Mutual Information $(I)$, or transinformation, of two random variables is a quantity measuring the mutual dependence of the two variables [24], [25]. In our case, the objective is to find important descriptors as well as to quantify this importance. In [26], authors demonstrate that the mutual information between gaussian (normaly distributed) and multinomial (discrete) variables can be computed as indicated by equation 2 . For this equation, it is assumed that: $C$ is a multinomial random variable with $r$ possible values and a probability distribution given by $P(C=c)=P(c) ; X$ is a random variable with a normal density function of parameters $\mu$ et $\sigma^{2} ; X$ conditioned to $C=c$ follows a normal density function with parameters $\mu_{c}$ et $\sigma_{c}{ }^{2}$.

$$
I(X ; C)=\frac{1}{2}\left[\log \left(\sigma^{2}\right)-\sum_{c=1}^{r} P(c) \log \left(\sigma_{c}^{2}\right)\right]
$$

This equation can yet be reduced to the form of the equation 3 .

$$
I(X ; C)=\log (\sigma)-\sum_{c=1}^{r} P(c) \log \left(\sigma_{c}\right)
$$

In the case that $X$ is a discrete variable with $q$ modalities, $I$ can be computed with equation 4 .

$$
I(X ; C)=\sum_{x=1}^{q} \sum_{c=1}^{r} P(x, c) \log \left(\frac{P(x, c)}{P(x) P(c)}\right)
$$

So, $I$ can be computed for all variables (descriptors) of the industrial system. The most important variables for the classification task will be those having an important $I$ value.

\section{NEW PROCEDURE FOR FAULT DIAGNOSIS}

The main idea of the procedure we are proposed for fault diagnosis in industrial processes with bayesian network classifier is described on figure 3 .

Like each data-driven technique, this procedure requires a database, and more particularly a database of faults. This database contains examples of each fault that we want to diagnosis. So, for each recorded fault, we have the type of the fault and the value of the different variables. At this time, mutual information between class node $\mathrm{C}$ (variable which codes the type of fault) and each descriptor $X_{i}$ (variable of the process) can be computed. So, we can sort the variables in decreasing order of mutual information. This is the step of variables selection. For this step, a threshold value $\tau$ is used. This threshold value must be fixed at $k \times \max \left(I\left(X_{i} ; C\right)\right)$ with $0<k<1$. Based on past experiences, we can advice a value of $k$ around 0.3 . If we take

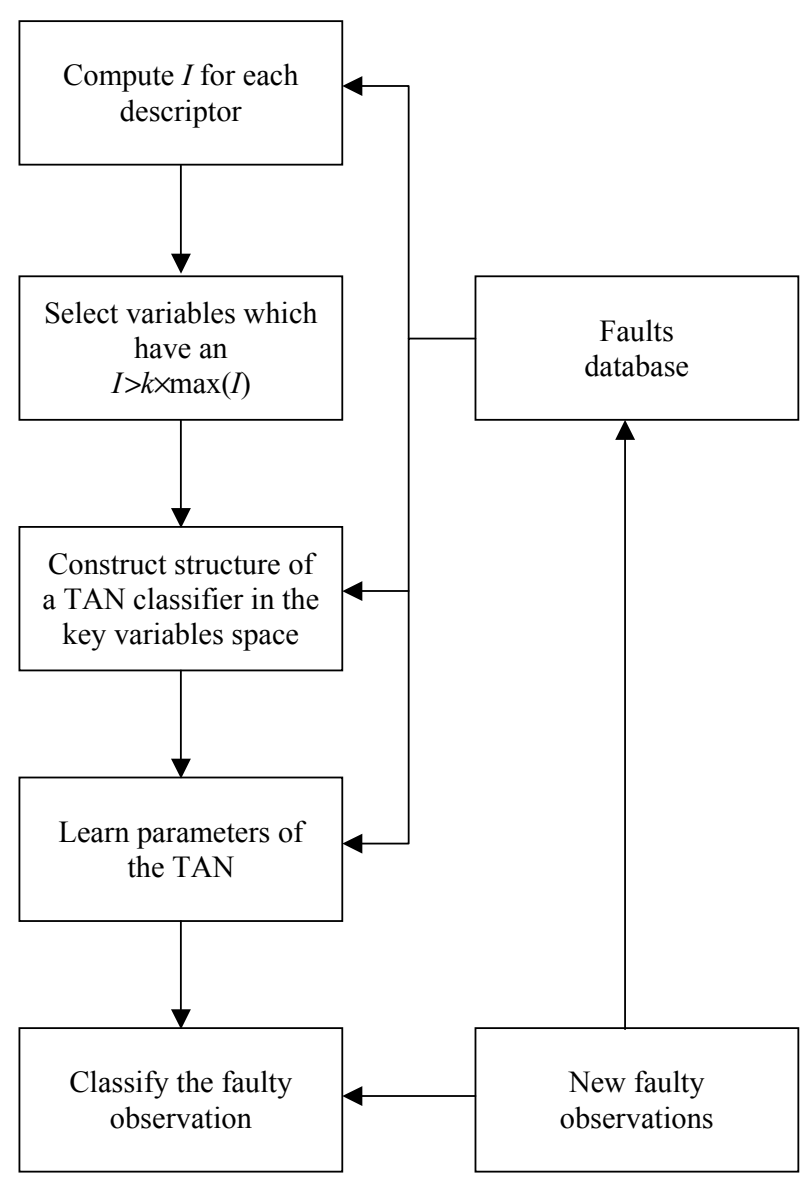

Fig. 3. Fault diagnosis procedure

lower value of $k$, we will select more key variables, but with more general information. Otherwise, if we take higher value of $k$, the number of key variables will be reduced, but we will have less general information. In conclusion, each variable with mutual information less than $\tau$ is eliminated. So, we exclusively keep variables giving important information for the discrimination of the classes.

After that, we have to construct our classifier. We can construct a naïve bayesian network (bayes classifier). But, as we already explained in the previous section, this classifier makes the assumption that all descriptors are statistically independent which is not the case in many complex industrial processes. So, on the faults database, we construct the structure of the TAN classifier by using only key variables. After the construction of the TAN, the parameters of this classifier can be estimated, from the faults database, with the MLE method. Only then after, we can classify a new faulty observation. If engineers or operators give the confirmation of the diagnosis of the system, the faulty observation and his class are added to the faults database.

In the next section, an application of this procedure is made on a benchmark problem: the Tennessee Eastman Process (figure 4). 


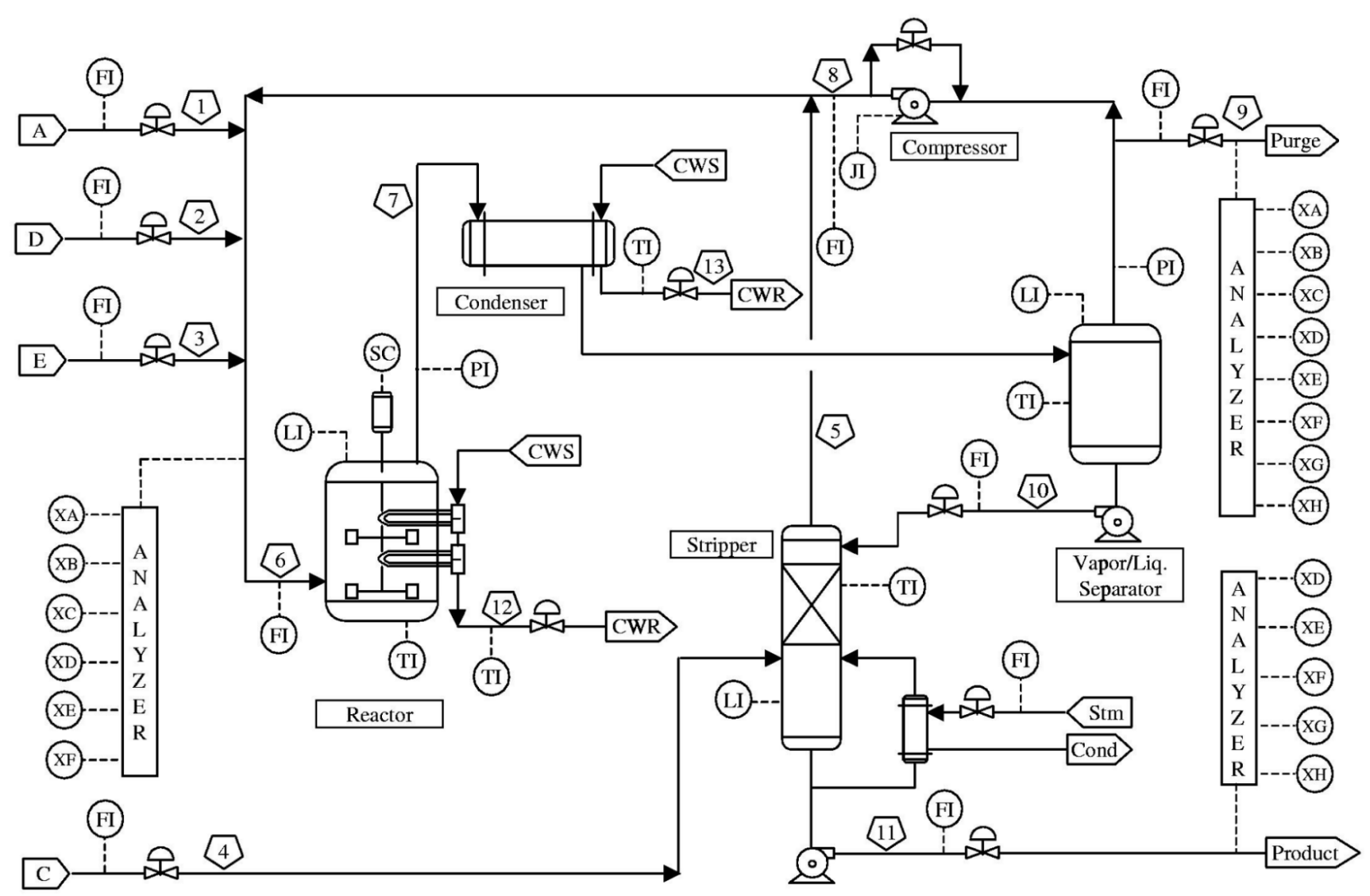

Fig. 4. Process flowsheet of the TEP

\section{Application to the TEP}

The process simulator for the Tennessee Eastman Industrial Challenge Problem was created by the Eastman Chemical Company to provide a realistic industrial process in order to evaluate process control and monitoring methods [27]. The Tennessee Eastman Process (TEP) is a chemical process. It is composed of five major operation units: a reactor, a condenser, a compressor, a stripper and a separator. Four gaseous reactant A, C, D, E and an inert $\mathrm{B}$ are fed to the reactor where the liquid products F, G and $\mathrm{H}$ are formed. This process has 12 input variables and 41 output variables. It has 20 types of identified faults.

The TEP is entirely described in the article of Downs and Vogel [27]. A computer simulation of this process was implemented on Matlab by Ricker [28]. This plant is openloop unstable. So, it is also a benchmark problem for control techniques. Some fault detection approaches have been tested on the TEP [8], [29], [30]. Some fault diagnosis techniques have also been tested on the TEP [11], [31], [32], [33] with the plant-wide control structure recommended in Lyman and Georgakis [34]. In [31], [32], [33], authors focus on only 3 types of faults and give the datasets they used. For this reason, we will take the same data that in these articles and we will compare our approach to others.

Consequently, we have taken into account 3 types of faults named: fault 4, 9 and 11 (see table I). These three types of fault are good representations of overlapping data

\begin{tabular}{clcc}
\hline Class & Fault type & $\begin{array}{c}\text { Train } \\
\text { data }\end{array}$ & $\begin{array}{c}\text { Test } \\
\text { data }\end{array}$ \\
\hline 1 & $\begin{array}{l}\text { Fault 4: step change in the } \\
\text { reactor cooling water inlet } \\
\text { temperature }\end{array}$ & 480 & 800 \\
& $\begin{array}{l}\text { Fault 9: random variation } \\
\text { in D feed temperature }\end{array}$ & 480 & 800 \\
& $\begin{array}{l}\text { Fault 11: random varia- } \\
\text { tion in the reactor cooling } \\
\text { water inlet temperature }\end{array}$ & 480 & 800 \\
\hline
\end{tabular}

TABLE I

Description of FAUlt DATASETS

and so, are not easy to classify.

For each type of faults, we have 2 datasets: a training sample and a testing sample, containing respectively 480 and 800 observations as indicated on the table I. All computations implicating bayesian network have been made on Matlab with the BNT (BayesNet Toolbox) developed by Murphy [35]. For an objective comparison to other methods, new faulty observations were not added to the faults database, like it was mentioned in the figure 3 . The faults database is composed of the 3 training samples.

Firstly, mutual information is computed for all variables. 
The results of this computation are given in the figure 5 . By using a $k$ value of 0.25 , the threshold value $\tau$ will be 0.27 , and we can see on figure 5 that variables 9 (reactor temperature) and 51 (reactor cooling water valve position) are the important variables for discriminating the different types of fault. Chiang in [32] has obtained the same conclusion with different methods (contribution charts, discriminant partial least square, genetic algorithms combined with Fisher discriminant analysis). So, the new variables space is defined by this 2 variables: 9 and 51 .

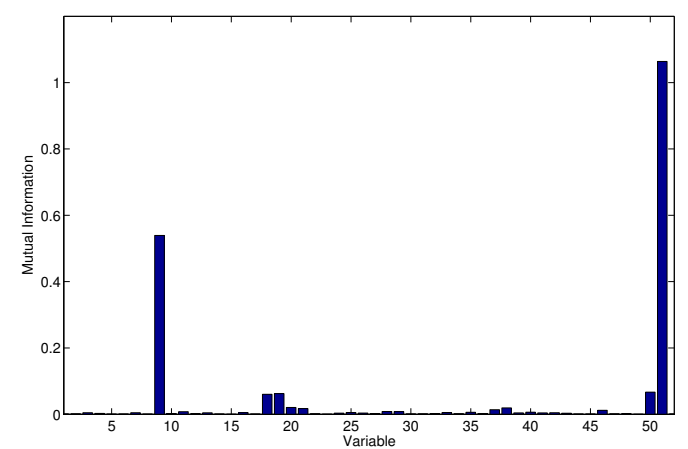

Fig. 5. Mutual Information for the TEP application

After that, we can construct the TAN classifier which will be a NBN with an edge between variable 9 and variable 51 , in order to take into account the correlation between these variables (correlation coefficient between these 2 variables is approximatively equal to 0.42 ). Parameters of this classifier are computed with the MLE method. This classifier is drawn on figure 6 .

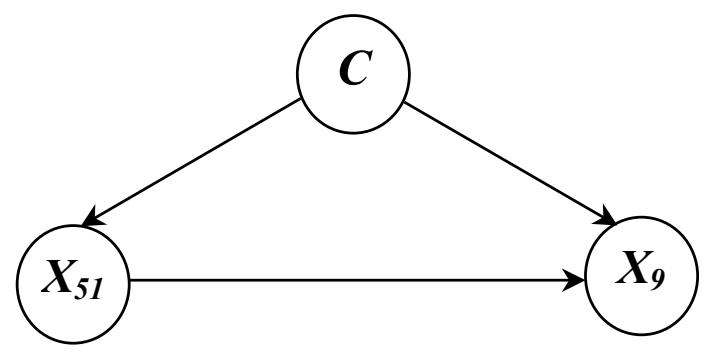

Fig. 6. TAN for the TEP

At this moment, we can classify the test sample with this classifier (2400 observations to classify). The results of this classification are given in the table II. The number given is the misclassification rate, which is the percentage of observations which are not well classified. We are also giving the results of other methods on the same data. We notify that the results for the FDA (Fisher Discriminant Analysis), SVM (Support Vector Machines), PSVM (Proximal Support Vector Machines) and ISVM (Independent Support Vector Machines) methods are extracted from [32] and [33]. We also have computed the misclassification rate that would be obtained by the use of NBN.

In the table II, we can see that the TAN outperforms all other methods. The fact that TAN outperforms FDA is

\begin{tabular}{cc}
\hline Method & Misclassification rate \\
\hline FDA & $16 \%$ \\
PSVM & $6.0 \%$ \\
SVM & $6.5 \%$ \\
ISVM & $6.0 \%$ \\
NBN & $7.8 \%$ \\
TAN & $\mathbf{5 . 9 \%}$ \\
\hline
\end{tabular}

TABLE II

RESULTS OF THE APPLICATION

not surprising because FDA is a linear technique compared with TAN which is quadratic. This higher performance can be explained by the fact that the TAN takes into account the strong correlation between variables 9 and 51 (correlation of 0.42 ). We can see that the results obtained by using the TAN are quite similar to SVM based techniques (SVM, PSVM, ISVM), but obtained with less computation effort.

We can see on figure 7 that the different classification areas of the classifier are fitting well the data shape and that the fault classes are normally distributed (ellipsoidal shapes). The results of the TAN would not be so efficient if the data was not normal.

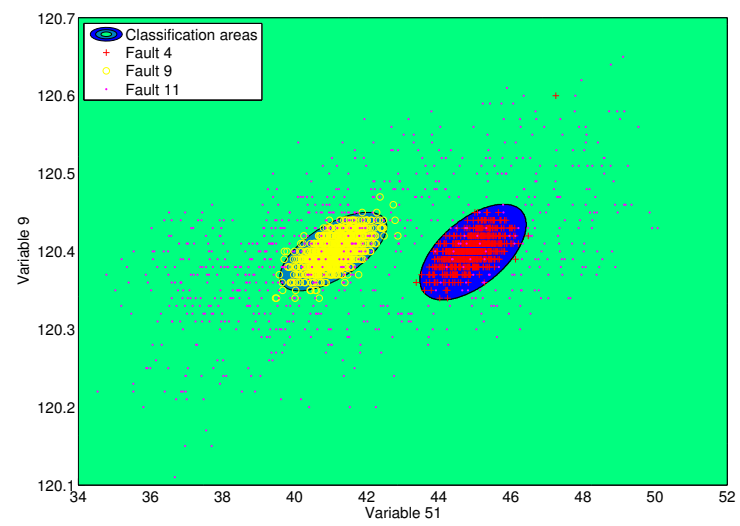

Fig. 7. Classification areas of the TAN

An interesting way to deal with not normally distributed variables will be the gaussian mixture model. This model can be a good answer to the problem of normally distributed data assumption.

The major drawback of this new procedure is that the classification is done in the reduced space of variables. So, if a new type of fault (not present in the process evolution history) appears and this new fault type is detectable only on variables which are not selected like key variables, the procedure can not be able to diagnosis the apparition of this new fault. 


\section{Conclusions And OUtLoOKs}

The main interest of this article is the application of a new procedure for fault diagnosis of industrial processes. This procedure uses a faults database for computing mutual information between each descriptor and the class variable. So, we can identify important variables for discrimination of the different types of faults. The classification space is reduced to these key variables. Also funded on the faults database, a Tree-Augmented bayesian Network is constructed and his parameters are learnt from the database. Performances of this approach were tested on a concrete example: the Tennessee Eastman Process. Results of this method are quite good and outperforms some previous results of other methods.

Outlooks of this procedure will be application of similar techniques, based on bayesian networks, in order to improve the diagnosis of the faults (cases of a non identified fault, case of non normally distributed data). We intent to study also in which way the fault detection step can be made with a bayesian network in order to have an homogeneous method for the fault detection and diagnosis.

\section{Acknowledgments}

This work was supported by a $\mathrm{PhD}$ purpose grant from "Angers Loire Métropôle" for the first author.

\section{REFERENCES}

[1] D. C. Montgomery, Introduction to Statistical Quality Control, Third Edition. John Wiley and Sons, 1997.

[2] W. A. Shewhart, Economic control of quality of manufactured product. New York: D. Van Nostrand Co., 1931.

[3] S. W. Roberts, "Control chart tests based on geometric moving averages," Technometrics, vol. 1, pp. 239-250, Aot 1959.

[4] H. Hotelling, "Multivariate quality control," Techniques of Statistical Analysis, pp. 111-184, 1947.

[5] J. Westerhuis, S. Gurden, and A. Smilde, "Standardized qstatistic for improved sensitivity in the monitoring of residuals in mspc," Journal of Chemometrics, vol. 14, no. 4, pp. 335-349, 2000.

[6] E. J. Jackson, "Multivariate quality control," Communication Statistics - Theory and Methods, vol. 14, pp. 2657 - 2688, 1985.

[7] B. R. Bakshi, "Multiscale pca with application to multivariate statistical process monitoring," AIChE Journal, vol. 44, no. 7, pp. 1596-1610, 1998.

[8] M. Kano, K. Nagao, S. Hasebe, I. Hashimoto, H. Ohno, R. Strauss, and B. Bakshi, "Comparison of multivariate statistical process monitoring methods with applications to the eastman challenge problem," Computers and Chemical Engineering, vol. 26 , no. 2 , pp. 161-174, 2002.

[9] R. L. Mason, N. D. Tracy, and J. C. Young, "Decomposition of $\mathrm{t} 2$ for multivariate control chart interpretation," Journal of Quality Technology, vol. 27, no. 2, pp. 99-108, 1995.

[10] R. Mason, N. Tracy, and J. Young, "A practical approach for interpreting multivariate t2 control chart signals," Journal of Quality Technology, vol. 29, no. 4, pp. 396-406, 1997.

[11] L. H. Chiang, E. L. Russell, and R. D. Braatz, Fault detection and diagnosis in industrial systems. New York: Springer-Verlag, 2001.

[12] J. Pearl, Probabilistic Reasoning in Intelligent Systems: Networks of Plausible Inference. Morgan Kaufmann Publishers, 1988.

[13] E. Charniak, "Bayesian networks without tears," AI Magazine, vol. 12 , no. 4 , pp. 50-63, 1991.

[14] F. V. Jensen, An introduction to Bayesian Networks. Taylor and Francis, London, United Kingdom, 1996.

[15] P. Langley, W. Iba, and K. Thompson, "An analysis of bayesian classifiers," in National Conference on Artificial Intelligence, 1992.
[16] N. Friedman, D. Geiger, and M. Goldszmidt, "Bayesian network classifiers," Machine Learning, vol. 29, no. 2-3, pp. 131-163, 1997.

[17] P. Domingos and M. J. Pazzani, "Beyond independence: Conditions for the optimality of the simple bayesian classifier," in International Conference on Machine Learning, 1996.

[18] I. Inza, P. Larranaga, B. Sierra, R. Etxeberria, J. Lozano, and J. Pena, "Representing the behaviour of supervised classification learning algorithms by bayesian networks," Pattern Recognition Letters, vol. 20, no. 11-13, pp. 1201-1209, 1999.

[19] M. G. Madden, "The performance of bayesian network classifiers constructed using different techniques," in Proceedings of European Conference on Machine Learning, Workshop on Probabilistic Graphical Models for Classification, September 2003.

[20] Y. Yang and G. I. Webb, "A comparative study of discretization methods for naive-bayes classifiers," in Proceedings of PKAW 2002, 2002.

[21] C. Chow and C. Liu, "Approximating discrete probability distributions with dependence trees," Information Theory, IEEE Transactions on, vol. 14, no. 3, pp. 462-467, 1968.

[22] M. Sahami, "Learning limited dependence bayesian classifiers," in Second International Conference on Knowledge Discovery in Databases, 1996.

[23] I. Kononenko, "Semi-naive bayesian classifier," in EWSL-91: Proceedings of the European working session on learning on $M a$ chine learning, pp. 206-219, 1991.

[24] C. E. Shannon, "A mathematical theory of communication," Bell Sys. Tech. J., vol. 27, pp. 379-423, 623-656, 1948.

[25] T. M. Cover and J. A. Thomas, Elements of Information Theory. John Wiley and Sons, 1991.

[26] A. Perez, P. Larranaga, and I. Inza, "Supervised classification with conditional gaussian networks: Increasing the structure complexity from naive bayes," International Journal of Approximate Reasoning, vol. In Press, Corrected Proof, pp. -, 2006.

[27] J. Downs and E. Vogel, "Plant-wide industrial process control problem," Computers and Chemical Engineering, vol. 17, no. 3, pp. 245-255, 1993.

[28] N. Ricker, "Decentralized control of the tennessee eastman challenge process," Journal of Process Control, vol. 6, no. 4, pp. 205$221,1996$.

[29] J.-M. Lee, C. Yoo, and I.-B. Lee, "Statistical monitoring of dynamic processes based on dynamic independent component analysis," Chemical Engineering Science, vol. 59, no. 14, pp. 2995$3006,2004$.

[30] U. Kruger, Y. Zhou, and G. Irwin, "Improved principal component monitoring of large-scale processes," Journal of Process Control, vol. 14, no. 8, pp. 879-888, 2004.

[31] L. Chiang, E. Russell, and R. Braatz, "Fault diagnosis in chemical processes using fisher discriminant analysis, discriminant partial least squares, and principal component analysis," Chemometrics and Intelligent Laboratory Systems, vol. 50, no. 2, pp. 243-252, 2000.

[32] L. Chiang, M. Kotanchek, and A. Kordon, "Fault diagnosis based on fisher discriminant analysis and support vector machines," Computers and Chemical Engineering, vol. 28, no. 8, pp. 1389-1401, 2004.

[33] A. Kulkarni, V. Jayaraman, and B. Kulkarni, "Knowledge incorporated support vector machines to detect faults in tennessee eastman process," Computers and Chemical Engineering, vol. 29, no. 10, pp. 2128-2133, 2005.

[34] P. Lyman and C. Georgakis, "Plant-wide control of the tennessee eastman problem," Computers and Chemical Engineering, vol. 19, no. 3, pp. 321-331, 1995.

[35] K. P. Murphy, "The bayes net toolbox for matlab," in In Computing Science and Statistics : Proceedings of Interface, 2001. 